\title{
Le programme et son exécution
}

\author{
Axel Kahn
}

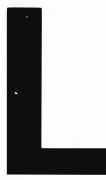

$\triangle$ inti

intérêt scientifique et les possibles retombées médicales, voire, plus généralement, biotechnologiques du programme Génome Humain sont peu contestables et, en définitive, peu discutés. On peut identifier, parmi les objectifs raisonnables de cet effort collectif, la localisation et la caractérisation des gènes de maladie et la découverte de nouveaux gènes dont le pouvoir codant orientera vers une fonction probable qu'il sera possible par les moyens actuels (incluant la recombinaison homologue ou la transgénèse) de tester. Cette nouvelle démarche, la seule qui mérite vraiment l'appellation de "génétique inverse " (puisqu'elle va du gène à la fonction... et non de la fonction au gène), est sans aucun doute de nature à accélérer considérablement l'identification des mécanismes génétiques du contrôle des grands événements et des grandes fonctions biologiques : développement, différenciation, sénescence, fonctionnement des organes et des systèmes de communication intercellulaires. Le systématisme de l'approche, s'adressant au supposé inutile aussi bien qu'au probablement significatif, a aussi le considérable avantage d'inclure l'imprévisible dans les buts poursuivis, c'est-à-dire ce futur incertain des sciences qui sous-tend la part de rêve si nécessaire en des temps où la recher-

\author{
Axel Kahn \\ Rédacteur en chef. Directeur de l'U. 129 de \\ l'Inserm. \\ ICGM, Inserm U. 129, 24, rue du Faubourg \\ Saint-Jacques, 75014 Paris, France.
}

che devient souvent fonction d'une unité qui serait l'année $\mathrm{x}$ chercheur $\mathrm{x}$ millions de dollars investis.

Les scientifiques doivent cependant prendre conscience de ce que, par son ampleur, sa nouveauté, son objet et l'utilisation possible de ses résultats, ce programme ne créera pas que de la connaissance, mais aussi une multitude d'outils nouveaux dont il faudra bien réglementer l'utilisation sociale et commerciale, et une gigantesque confusion sur la nature du "programme génétique " ainsi élucidé.

\section{Connaissance et propriété intellectuelle}

La question de la propriété intellectuelle des séquences d'ADN a été récemment mise en lumière, de manière éclatante, par la tentative du $\mathrm{NIH}$ de prendre des brevets sur des séquences partielles d'ADNc $\left(\mathrm{m} / \mathrm{s} n^{\circ} 9\right.$, vol. 7 p. 960), la critique revenant le plus souvent étant que " l'on ne savait pas même ce que faisaient, ce qu'étaient les gènes auxquels appartenaient ces éléments de séquence ". De fait, il y a bien longtemps que plus personne parmi les artisans du génome ne conteste plus la prise de brevet sur des gènes identifiés, couvrant tout ce à quoi l'on imagine qu'ils pourraient servir: diagnostic prénatal, thérapie génique, utilisation biotechnologique, etc. Autant la protection de procédés nécessitant la connaissance du gène étudié rentre tout à fait dans la logique traditionnelle des brevets, autant l'extension préventive de leur champ d'application à toutes les utilisations envisageables de ce gène... envisageables mais non prouvées, est de nature toute différente. Dans ce cas, en effet, la seule "invention " réellement brevetée, c'est la découverte du gène, l'élucidation de sa séquence et de sa fonction, c'est-à-dire l'accession à la connaissance d'un objet pré-existant à l'intervention du scientifique. Il s'agit donc bien là, en réalité exactement au même titre que dans le cas des séquences partielles du NIH, d'une appropriation d'une connaissance acquise du monde naturel, accompagnée de vagues spéculations sur sa possible utilisation commerciale.

\section{La maîtrise de l'utilisation des nouveaux outils créés par les études génétiques}

Une des plus évidentes caractéristiques du programme Génome est qu'il crée des outils dont le champ d'application est l'identité génétique et l'intimité physiologique de l'homme : empreintes génétiques et leurs remarquables capacités d'identification et de précision des filiations, détection du sexe génétique et d'un nombre croissant de prédestinations ou de prédispositions à des maladies ou à des caractéristiques non pathologiques. Parce que ces moyens nouveaux posent des problèmes légaux (définition des règles de filiation, utilisation dans des procès) ou bien sont de nature à fournir des informations qui peuvent être un formidable levier de puissance pour ceux qui les possèdent et une puissante circonstance d'exclusion, d'assujettissement et de déstabilisation psychologique pour ceux sur qui elles portent, beaucoup sentent bien que leur emploi devait être mâ̂trisé. En France, l'accession aux empreintes génétiques fera l'objet 
d'une loi qui sera discutée au printemps de cette année. Dans le même temps, cependant, nous est donné un remarquable exemple des mécanismes qui régiront l'utilisation de ce nouvel arsenal technologique si un effort collectif de la société, éventuellement stimulée et informée par ses scientifiques, n'est pas consenti pour en assurer la maîtrise : les lois du marché. Le marché n'est ni bon, ni mauvais, il a sa logique qui est... d'exister ou de se créer. Par conséquent, si une entreprise de biotechnologie se crée, dont la raison d'être est de vendre du diagnostic génétique, elle vendra naturellement tout ce qui peut l'être et dont la commercialisation n'est pas interdite ou réglementée, obéissant ainsi simplement à des règles dont dépend son succès, voire sa survie. En matière de recours aux tests génétiques, la réussite ou l'échec d'une telle conquête d'un marché a donc toutes les chances de remplacer la réflexion de la société dans l'établissement des usages. C'est ce qui vient de se produire concernant l'adoption par le comité international olympique $(\mathrm{CIO})$ du test de détection du gène de détermination du sexe, $S R Y$, comme moyen de vérification de la féminité des athlètes femmes. D'un côté, une instance sportive ayant un problème technique à résoudre (l'équité des épreuves) et se trompant sur sa nature, de l'autre, une toute jeune société dont l'avenir dépend des marchés qu'elle est capable de créer, dans le contexte d'une absence de réflexion collective véritable sur la signification profonde des questions posées et des pseudo-réponses apportées par les tests génétiques proposés. A l'arrivée, la première utilisation non médicale et non judiciaire d'un test génétique destiné à vérifier le classement correct d'êtres humains dans des catégories dont les limites sont passablement incertaines, sans souci des conséquences psychologiques pour l'individu de la révélation d'une identité génétique discordante avec l'identité apparente et acceptée. Les procédures décisionnelles ayant fonctionné dans cette affaire montrent à l'évidence que, sans rationalisation sociale de ce commerce-là, on pourra voir demain l'achat de tests génétiques d'aptitude à des performances diverses par des employeurs, de tests de susceptibilité à toute une série d'affections par les assureurs, c'est-àdire l'accession de petits groupes à des informations sur l'individu de nature à déséquilibrer gravement ce que devraient être des rapports entre hommes libres.

\section{Les illusions créées par le terme de " programme génétique "}

Il est répété à l'envie par les vulgarisateurs du programme Génome, ainsi que par certains de ses praticiens, que son but est la connaissance du patrimoine héréditaire humain, du programme génétique : les prédispositions, les prédestinations, les susceptibilités... et l'écho du public ajoute "tout, quoi...". Ce "tout " englobe la définition de l'Être humain et son destin. Il s'agit là, naturellement, d'une gigantesque confusion alimentće par une absence de toute interrogation sur ce que représente, dans le champ du vivant, au sein du monde animal, un Homme. La définition positive de la personne humaine a fait l'objet de tant d'études qu'il n'est pas question, ici, de s'y essayer ( $m / s n^{\circ} 6$, vol. 7 p. 602). Elle procède sûrement en partie des remarquables aptitudes mentales de l'Homme, sans se limiter à celles-ci au niveau individuel (sinon on pourrait considérer... comme jusqu'en 1945 assez largement en Europe et aux États-Unis, que les personnes n'ayant pas (toutes) ces capacités mentales ne sont pas des êtres humains !) Or, la principale caractéristique du cerveau humain est sa remarquable plasticité sur laquelle plusieurs articles et nouvelles, ainsi que le lexique de neurobiologie de $m / s$, ont insisté. En d'autres termes, le cerveau humain peut être largement considéré comme un organe fabriqué par l'expérience du vécu, les interactions avec l'environnement matériel, intellectuel et affectif. Cette plasticité a un substratum neuroanatomique et physiologique que l'on commence à connaîtrc : la stabilisation sélective ou l'établissement de circuits neuronaux. Certes, les propriétés globales de malléabilité du cerveau sont la conséquence du programme génétique humain, mais elles ne déterminent pas plus la nature de l'organe fini (il n'est, en réalité, jamais fini, conservant jusqu'à la mort une certaine plasticité), que la matière première que sculpte l'artiste ne permet de prévoir l'œuvre. L'enchaînement complet des trois milliards de paires de bases de notre génome dit, par conséquent, bien peu de l'Humain. Le " programme " dont il est question, quand on parle de programme génétique, n'est donc que celui qui va déterminer les propriétés brutes de la matière vivante, toutes ses propriétés mais rien qu'elles, le reste étant heureusement du domaine de l'imprévisibilité. Par ailleurs, tout biologiste sait que l'exécution fidèle d'un programme ne dépend jamais d'une seule information mais est la résultante d'une combinatoire plus ou moins complexe. Ainsi, pour en revenir à l'exemple du test génétique chez les athlètes, la présence d'un gène $S R Y$ ne signifie évidemment pas que l'individu a des caractéristiques physiques, notamment des performances sportives, mâles : ce gêne peut être inactif, la sécrétion des hormones mâles peut être qualitativement ou quantitativement anormale, les récepteurs de ces hormones peuvent être absents ou non fonctionnels, etc. En définitive, seules comptent ici la sécrétion et l'action biologique de la testostérone, faciles à explorer par des dosages hormonaux et un examen physique. La présence du gène $S R Y$ est un élément important de la définition du sexe. Il ne saurait la résumer et n'est qu'indirectement lié à l'exigence de l'équité des compétitions sportives... qui n'est qu'un problème d'hormones. Naturellement, SRY est, si l'on peut dire, encore plus insuffisant pour rendre compte de la féminité... c'est-à-dire de la "femellité " dans l'espèce humaine. Celle-ci, outre ses composantes génétiques et hormonales, a aussi des dimensions psychologiques et sociales (ou légales) qui peuvent être en discordance avec les précédentes comme l'illustre, notamment, le cas des transsexuels.

\section{Conclusion}

J'ai voulu, dans cette brève analyse, attirer l'attention des scientifiques engagés dans cette grande et belle aventure du déchiffrage du génome sur leurs responsabilités, qui ne s'arrêtent pas au seuil de leurs laboratoires : ils créent non seulement de la connaissance et de l'espoir, mais aussi, comme bien sou- 
vent dans l'histoire du progrès, des outils potentiellement redoutables en ce qu'ils s'adressent à notre identité et à notre intimité génétiques. Ils fondent également les bases pseudo-scientifiques d'un eugénisme moderne réduisant, peu ou prou, l'Humain à son programme génétique. Conscients du pouvoir des méthodes et du caractère intellectuellement dévastateur des illusions engendrées, les chercheurs doivent jouer tout leur rôle pour informer la société et participer à la maîtrise de l'utilisation des possibilités techniques qu'ils ont contribué à créer

TIRÉS A PART

A. Kahn. 